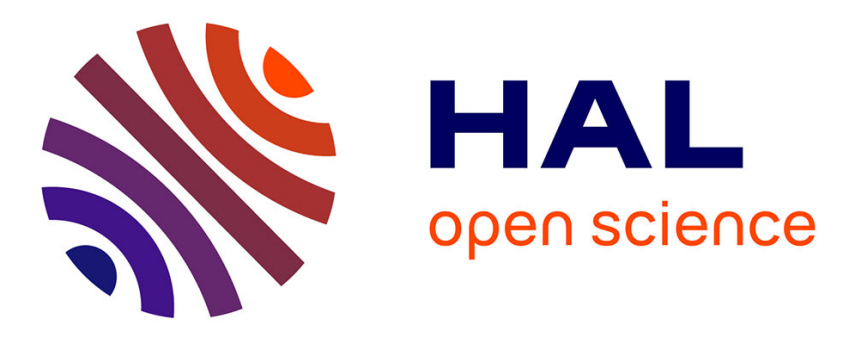

\title{
Mode Coupling Measurement in Dual-Frequency Quantum Well-based VECSEL
}

Gaëlle Brévalle, Salvatore Pes, Cyril Paranthoen, Mathieu Perrin, Christophe

Levallois, Cyril Hamel, Alexandru Mereuta, Andrei Caliman, Eli Kapon, Laurent Chusseau, et al.

\section{To cite this version:}

Gaëlle Brévalle, Salvatore Pes, Cyril Paranthoen, Mathieu Perrin, Christophe Levallois, et al.. Mode Coupling Measurement in Dual-Frequency Quantum Well-based VECSEL. Compound Semiconductor Week 2019 (CSW 2019), May 2019, Nara, Japan. pp.1-2. hal-02290594

\section{HAL Id: hal-02290594 https://hal.science/hal-02290594}

Submitted on 5 Nov 2021

HAL is a multi-disciplinary open access archive for the deposit and dissemination of scientific research documents, whether they are published or not. The documents may come from teaching and research institutions in France or abroad, or from public or private research centers.
L'archive ouverte pluridisciplinaire HAL, est destinée au dépôt et à la diffusion de documents scientifiques de niveau recherche, publiés ou non, émanant des établissements d'enseignement et de recherche français ou étrangers, des laboratoires publics ou privés. 


\title{
Mode Coupling Measurement in Dual-Frequency Quantum Well-based VECSEL
}

\author{
Gaëlle Brévalle ${ }^{1}$, Salvaore Pes ${ }^{1}$, Cyril Paranthoën ${ }^{1}$, Mathieu Perrin ${ }^{1}$, Christophe Levallois ${ }^{1}$, Cyril \\ Hamel $^{1}$, Alexandru Mereuta ${ }^{2}$, Andrei Caliman², Eli Kapon ${ }^{2}$, Laurent Chusseau ${ }^{3}$, Hervé Folliot ${ }^{1}$ and \\ Mehdi Alouini ${ }^{1}$ \\ ${ }^{1}$ Univ Rennes, INSA Rennes, CNRS, Institut FOTON - UMR 6082, F-35000 Rennes, France \\ ${ }^{2}$ Laboratory of Physics of Nanostructures, Ecole Polytechnique Fédérale de Lausanne, CH-1015 \\ Lausanne, Switzerland \\ ${ }^{3}$ IES, Université de Montpellier, CNRS, Montpellier, France \\ gaelle.brevalle@insa-rennes.fr
}

\begin{abstract}
We experimentally investigate the Lamb coupling constant C in InGaAlAs Quantum Wells active medium. An Optically-Pumped Vertical-External-Cavity Surface-Emitting Laser emitting at $1.54 \mu \mathrm{m}$ is designed to sustain the oscillation of two orthogonally polarized modes sharing the same active region while separated by $1 \mathrm{~mm}$ in the rest of the cavity. It enables to tune independently the two wavelengths and to apply differential losses in order to directly measure the coupling constant. We found $C$ to be almost constant and equal to $0.839 \pm 0.023$ for a wavelength difference between the two eigenmodes ranging from $0.36 \mathrm{~nm}$ up to $10.8 \mathrm{~nm}$.
\end{abstract}

Keywords—Lamb Coupling; VECSEL; dual-frequency laser

\section{INTRODUCTION}

The Lamb coupling constant $\mathrm{C}$ governs the dynamics of two coupled modes in a laser, and consequently the dynamics of dual-frequency lasers. For $\mathrm{C}<1$, simultaneous oscillation of two modes is possible. In practical cases, this regime becomes increasingly perturbed as $\mathrm{C}$ approaches the unity, until moving towards bistability when $\mathrm{C}$ exceeds 1 [1]. In dual-frequency solid-state lasers, this coupling constant can be quite weak, $\mathrm{C} \sim 0.16$ [2], or high: $\mathrm{C} \sim 0.8$ [3] depending on the active medium. However, even though dual-wavelength oscillation has been demonstrated in semiconductor Quantum Well (QW) based Vertical External Cavity Surface Emitting Lasers (VECSELs) [4], a direct measurement of the Lamb coupling constant in QWs active media has never been reported. Indeed, because of the homogeneously broadened gain offered by QW-based active medium, the two eigenmodes of a QW-VECSEL suffer from strong coupling making it necessary to lift the degeneracy by introducing a spatial separation inside the active medium, thus reducing the coupling for the purpose of a steady dual-frequency oscillation. To the best of our knowledge, the value of such a coupling constant has only been measured by indirect methods based on the measurement of $\mathrm{C}$ for different spatial separations leading to an extrapolated value of $\mathrm{C}=0.8$ [5]. The purpose of our work is to measure directly this coupling constant for different wavelength separations between the two modes and to show possible dual-frequency operation without the necessity to reduce the coupling by introducing a spatial walk-off in the active medium.

\section{LAMB COUPLING CONSTANT MEASUREMENTS}

To that end, an Optically-Pumped Vertical-External-Cavity Surface-Emitting Laser (OP VECSEL) emitting at $1.54 \mu \mathrm{m}$ was designed to sustain the oscillation of the two orthogonally polarized modes sharing the same active region while separated in the rest of the cavity. The laser cavity is depicted in Figure 1a). Inside the cavity, we inserted two etalons to independently tune the two eigenmodes $I_{1}$ and $I_{2}$ separated by $1 \mathrm{~mm}$ thanks to a birefringent crystal. The etalons ensure in addition single frequency oscillation of each eigenmode, confirmed with a Fabry-Perot interferometer and an Optical Spectrum Analyzer (OSA). Some acquired spectra are shown in figure $1 \mathrm{~b}$ ). To measure $\mathrm{C}$, we introduce for one mode weak extra optical losses with a razor blade mounted on a piezoelectric actuator. We then measure the intensity variations of $I_{1}$ and $I_{2}$ to obtain the cross- to self-saturation ratios $K_{12}$. This operation is repeated on the other mode in order to obtain $\mathrm{K}_{21}$ and finally $\mathrm{C}=\mathrm{K}_{12} \mathrm{~K}_{21}$. As shown in Fig.3, $\mathrm{C}$ has been measured for several wavelength differences $\Delta \lambda$ between the two modes, by fixing $\mathrm{I}_{1}$ at $1535.4 \pm 0.2 \mathrm{~nm}$ while changing $\mathrm{I}_{2}$ 's wavelength by rotating the etalon. We found that $\mathrm{C}$ is almost constant for $\Delta \lambda$ ranging from $0.36 \mathrm{~nm}$ up to $10.8 \mathrm{~nm}$, with a mean value of 0.839 . The overall standard deviation is 0.023 , leading to $\mathrm{C}=0.839 \pm 0.023$.

This work is supported by the IDYLIC ANR project (ANR-15-CE24-0034-01).

[1] M. Sargent et al., Laser physics. London : Addison-Wesley, 1974

[2] M. Brunel, et al., "Differential measurement of the coupling constant between laser eigenstates," Appl. Phys. Lett., vol. 70, no. 16, pp. 2070-2072, Apr. 1997.

[3] A. McKay et al., "Polarisation-mode coupling in (100)-cut Nd: YAG," Opt. Express, vol. 15, no. 25, pp. 16342-16347, 2007.

[4] G. Baili et al., "Experimental demonstration of a tunable dual-frequency semiconductor laser free of relaxation oscillations," Opt. Lett., vol. 34, no. 21, pp. 3421-3423, Nov. 2009.

[5] V. Pal et al., "Measurement of the coupling constant in a two-frequency VECSEL," Opt. Express, vol. 18, no. 5, p. 5008, Mar. 2010. 


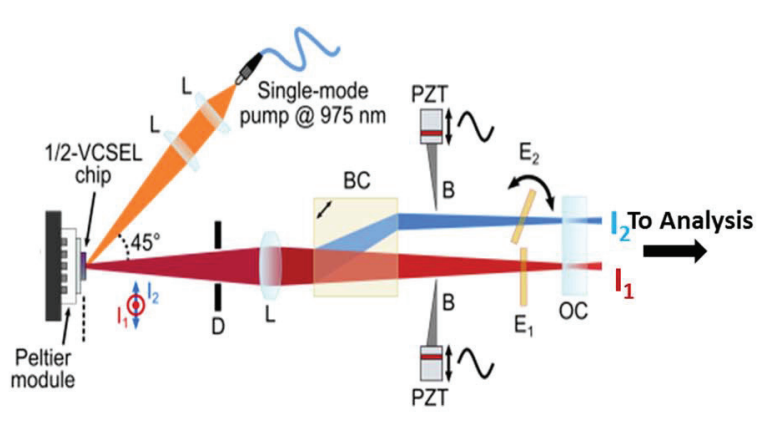

a)

Fig. 1. a) Coupling constant measurements cavity setup. L: lens, D: diaphragm, BC: YVO4 birefringent crystal, PZT: piezo actuator, B: razor blade, E: etalon, OC: planar output coupler. Index 1 and 2 are referred to the two orthogonally polarized eigenmodes $\mathrm{I}_{1}$ (ordinary) and $\mathrm{I}_{2}$ (extraordinary). b) Emission spectra acquired by the OSA for several wavelength differences.

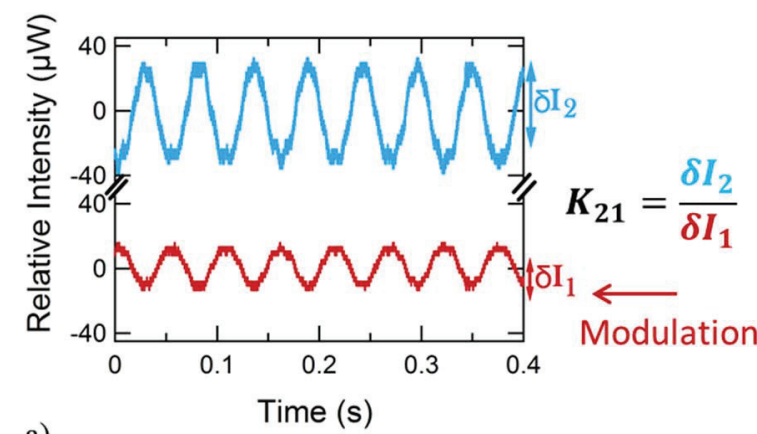

a)

Fig. 2. Evolution of the traces observed at the oscilloscope when the modulation losses are applied to a) mode $\mathrm{I}_{1}$ or $\mathrm{b}$ ) mode $\mathrm{I}_{2}$.

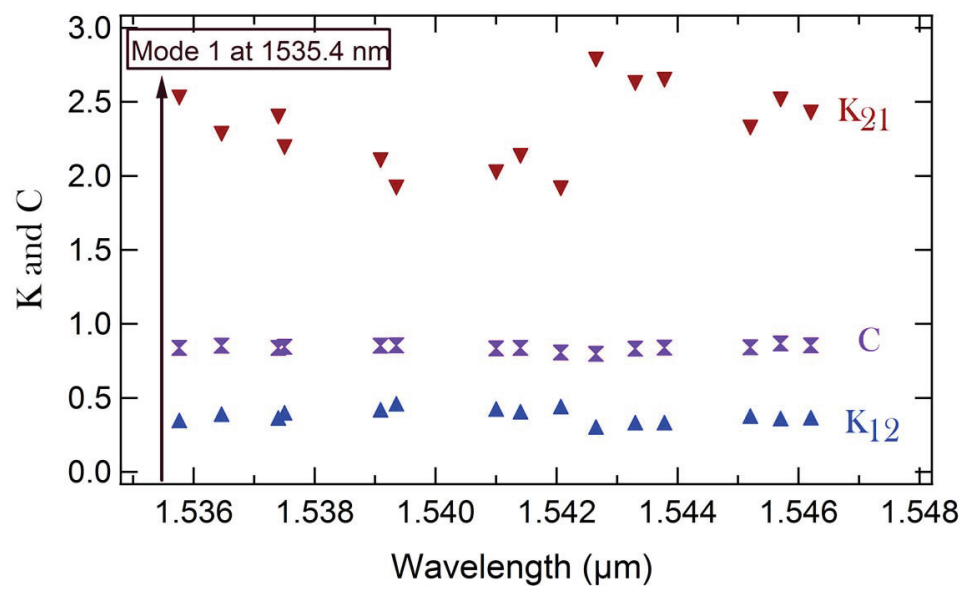

Fig. 3. Cross- to self- saturation ratios $K_{12}$ and $K_{21}$, and Lamb coupling constant $C$ versus the wavelength of mode $I_{2}$ with $I_{1}$ kept at $1535.4 \mathrm{~nm}$. 